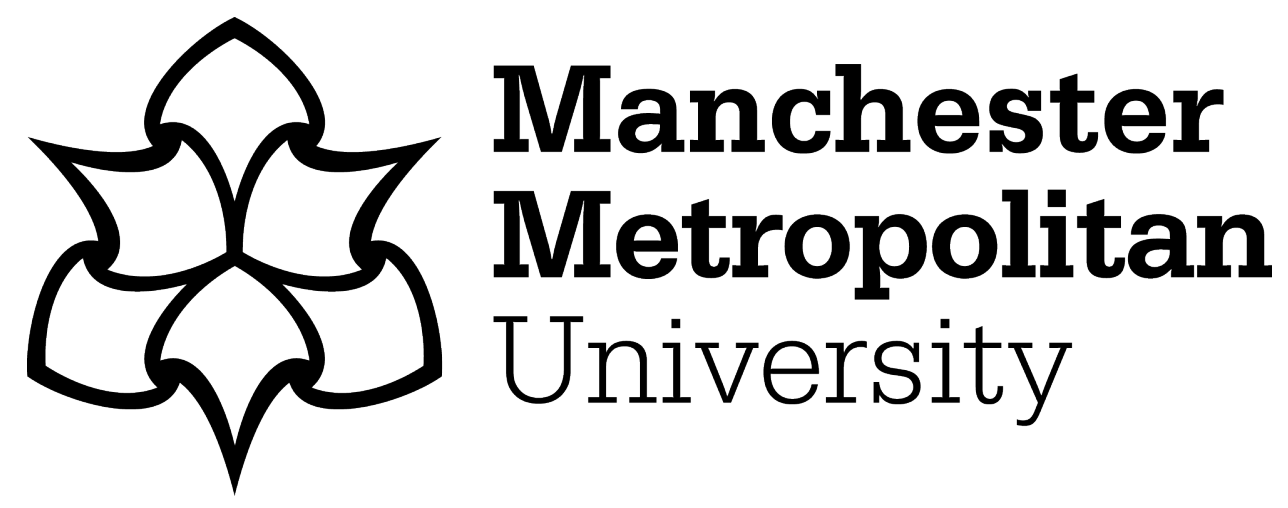

Gibson, L and Groom, Neil (2017) Ambiguity, manageability and the orchestration of organisational change: A case study of a Premier League Academy Manager. Sports Coaching Review, 7 (1). pp. 23-44. ISSN 2164-0629

Downloaded from: https://e-space.mmu.ac.uk/618323/

Version: Accepted Version

Publisher: Taylor \& Francis

DOI: https://doi.org/10.1080/21640629.2017.1317173

Please cite the published version 
$22 *$ Corresponding author: Luke Gibson, Sport, Outdoor \& Exercise Science,

23 Department of Life Sciences, College of Life \& Natural Sciences, University of AMBIGUITY, MANAGEABILITY AND THE ORCHESTRATION OF ORGANISATIONAL CHANGE: A CASE STUDY OF AN ENGLISH PREMIER LEAGUE ACADEMY MANAGER

\author{
${ }^{1}$ Luke Gibson* \\ ORCID: http://orcid.org/0000-0002-8401-8480 \\ ${ }^{1}$ University of Derby \\ Sport, Outdoor and Exercise Science
}

$\&$

ORCID: http://orcid.org/0000-0001-7633-3846

${ }^{2}$ Manchester Metropolitan University

Department of Exercise \& Sport Science

Sports Coaching Review

24 Derby, Buxton, SK17 6RY 1.gibson@derby.ac.uk

25

26 


\section{AMBIGUITY, MANAGEABILITY AND THE ORCHESTRATION OF ORGANISATIONAL CHANGE: A CASE STUDY OF AN ENGLISH PREMIER LEAGUE ACADEMY MANAGER}

\section{Abstract}

An academy is an organisational context operated by professional football clubs, governed by the rules of the English Football Association and the English Premier League. Academies provide coaching and education for youth football players aged from under 9 to under 21. The Academy Manager is responsible for the strategic leadership and operation of the club's academy. This includes implementing the club's philosophy, coaching and games programme, player education, and the management of academy staff. The purpose of this paper is to explore the experiences of Simon [pseudonym], an English Premier League Academy Manager, when implementing organisational change within an academy. Data were collected from a work-based diary and four in-depth semi-structured interviews. Wallace's (2003, 2004) notion of orchestration is used as an analytical frame to make sense of Simon's experiences through the change process and further our understanding of the social complexities of organisational change in elite sporting environments.

\section{Introduction}

Sports coaching can be understood to consist of a series of embedded, takenfor-granted, socio-cultural interactions that occur between social actors within a contextually bound environment (Cushion \& Jones, 2006, 2012; Groom, Cushion, \& Nelson, 2012; Jones \& Corsby, 2015). Indeed, recent empirical research in sports coaching has highlighted a number of negotiated, relational and complex realities associated with the everyday role of the coach (e.g. Nelson, Potrac, Gilbourne, 
1 Allanson, Gale, \& Marshall, 2013; Potrac \& Jones, 2009; Thompson, Potrac, \& Jones,

2 2015). The work of Potrac and Jones (2009), Potrac et al. (2013), Thompson et al.

3 (2013), and Nelson et al. (2013), among others, has highlighted that coaches,

4 particularly within the culture of football, have to deal with contradictory goals and

5 conflicting personal agendas, which are often driven by the structural vulnerabilities

6 associated with the working life of a coach. Importantly, this work has demonstrated

7 that coach education programmes have failed to provide coaching practitioners with the

8 knowledge and skills to deal with the complex, political and emotional realities

9 associated working with others in coaching contexts (Nelson et al., 2013; Potrac \&

10 Jones, 2009; Thompson et al., 2015).

11 Whilst early work has started to explore organisational structures in elite 12 football (e.g. Relvas, Littlewood, Nesti, Gilbourne, \& Richardson, 2010) and effective 13 and efficient change management processes in sport more broadly (e.g. Cruickshank \& 14 Collins, 2012a, 2012b, 2012c; Cruickshank, Collins, \& Minten, 2014), there is limited 15 empirical work from an interpretive perspective which reflects the social complexity 16 faced by elite level coaches, managers and sporting directors when implementing 17 organisational change. This is important, as there is a dearth of research examining the 18 motivations, actions and behaviours of coaches and other support staff to better 19 comprehend the complexities of practice (Cushion \& Jones, 2006, 2012; Groom et al., 20 2012; Santos, Jones, \& Mesquita, 2013). Therefore, this paper seeks to explore a 21 contemporary and original space within the sports coaching and sports management 22 literature - one that focuses upon the experiences and interactions of an English Premier 23 League Academy Manager whilst managing others (e.g. players, coaching staff, sports 24 science support staff, club directors, etc.) through the process of organisational change. 
To achieve this goal, Jones and Wallace (2005) have suggested that theorising regarding the management of complex educational change may provide a useful analytical frame to enhance our understanding of the realities and ambiguities of coaching and management, and thus better prepare practitioners for the realities of practice. Specifically, Jones and Wallace (2005) outlined the concept of orchestration as an innovative way of thinking about 'how to cope with uncontrollability, incomprehensibility, contradictory values and novelty as normal parts of everyday coaching life' (p. 128). Drawing upon Wallace's (2003) empirical findings examining the management of complex educational change, orchestration is described as behindthe-scenes string-pulling and the steering of complex change processes towards desired objectives, with constant evaluation and scrutiny to keep things going, and the maintenance of detailed oversight of the idiosyncrasies of each coaching situation (Jones, Bailey, \& Thompson, 2013). Therefore, the work of Wallace $(2003,2004)$ offers a novel analytical approach to understanding the social complexities of organisational change in elite sporting environments.

The significance of the study, then, rests in illuminating some of the everyday realities, actions and strategies of an elite coach working in the role of an English Premier League Academy Manager in order to provide a meaningful insight into the realities of working with a range of others. This work aims to open up a novel space to think about and better understand the role of the coach as a manager and social agent of organisational change. In doing so, the empirical grounding offered within the present study may start to provide a firmer foundation for the education of elite coaches entering into senior organisational managerial positions (e.g. Academy Manager, Technical Director, Head of Player Recruitment, and Sporting Director). 


\section{Research approach overview}

Case studies can be understood to be constructed in different ways depending

4 upon the subject, object, purpose, approach and process (Thomas, 2011). The subject

5 of the present study was a local knowledge case that had been appointed to implement

6 organisational change within an English Premier League Academy. Following a typical

7 interpretive case study approach, the object of the study - to explore the subjective

8 meanings, motivations and worldview that informed the participant's social actions -

9 emerged iteratively as inquiry progressed through the interview process (Thomas,

10 2011). The purpose of the case study was exploratory in nature, following an iterative

11 descriptive/illustrative approach. To make sense of the data, an analytical frame 12 (orchestration) was further introduced into the data analysis in an iterative manner

13 (Thomas, 2011). The case study process followed a single-participant combined 14 snapshot (diary) and retrospective (interview) design to explore the complexity of the 15 participant's day-to-day activities.

The interpretive case study: ontology and epistemology

The interpretive case study research design was rigorously developed, underpinned by ontological relativism (i.e. reality is multiple, created and mind dependent) and epistemological constructionism (i.e. knowledge is constructed and subjective), and analysed through a narrative analysis approach focusing upon the setting, characters and plot lines (Smith, 2013; Smith \& Sparkes, 2009; Smith, Sparkes,

23 \& Caddick, 2014). Thacher (2006) explained that, from an epistemological position,

24 the interpretive case study aims to illuminate the subjective meaning that people attach 25 to their actions: 'insofar as it focuses on values, it aims to describe the values currently 
1 held by the subjects of research' (p. 1635). The German sociologist Max Weber made

2 this distinction himself in his discussion of verstehen [a sociological approach towards

3 understanding the meaning of action from the point of view of the participant] (Thacher,

4 2006). Thacher (2006) further explained Weber's position that:

5

Social science should try to understand the meaning that action has for the people who engage in it, he clarified that "meaning" in this context referred to subjective meaning - most commonly, 'the actual existing meaning in the given concrete case of a particular actor.' Regardless, 'in no case does it refer to an objectively "correct" meaning or one which is "true" in some metaphysical sense'. (Thacher 2006, p. 1635)

Following the work of Weber, the ontological position of the interpretive case study is to contribute towards understanding (verstehen) through identifying the motivations and worldview that inform social action (Thacher, 2006). This is important because 'interpretive case studies offer explanations, since identifying the world or motives that lead people to behave in a particular way is also arguably a way of explaining their behaviour' (Thacher, 2006, p. 1635).

\section{Ethical approval, pilot work, and sampling procedure}

Prior to the investigation, institutional ethical approval was granted by the Life Sciences Research Ethics Committee at the University of Derby. Following the institutional ethical approval, the first author conducted a series of pilot interviews with two UEFA (Union of European Football Associations) qualified coaches to improve the process of enquiry (e.g. phrasing and contextual insight). Within this part of the 
1 research process, the initial lines of questioning regarding coach education, coaching

2 experience, and dealing with stakeholders as an academy coach were established. After

3 the pilot interviews were completed, the second author conducted a peer debrief of the

4 interviews and provided the first author with further guidance regarding the structure

5 of the questions and the use of elaboration and clarification probes (Spall, 1998).

6 A criteria-based purposive sampling strategy was utilised to identify potential 7 participants for the research project (Smith \& Sparkes, 2014). The participant inclusion

8 criteria were: (1) held the highest coaching award available (the UEFA Professional

9 Licence), (2) held the relevant qualification to manage an English Premier League 10 Category One Academy (the Academy Managers Licence), (3) had acted as both a 11 coach and Academy Manager within an English Premier League club, and (4) had been 12 through the process of implementing organisational change within an English Premier 13 League Academy.

14 Next, a shortlist of possible participants was created and discussed by the 15 research team. Once agreement had been reached, the second author contacted the 16 chosen participant who was already known to him through his previous work in elite 17 football. The second author explained to the participant the aims of the study, the 18 research process, and what would be required of him, and the participant subsequently 19 agreed to become the focus of the study.

21 The participant and context

22 The narrative is based upon the experiences of Simon (pseudonym), a forty23 three-year-old professional soccer coach, who at the time of the interviews was an 24 English Premier League Academy Manager working for an elite European club. Simon 25 was responsible for overseeing the development of youth soccer players aged between 
1 eight and twenty-one years. For Simon, this typically represented eighteen to twenty-

2 two players in a full-time coaching programme. His role as Academy Manager also

3 gave him responsibility for all coaches, players, scouts, physiotherapists, sport

4 scientists, administrators, and education and welfare officers involved within each age

5 group, from the Under 9s to the Under $21 \mathrm{~s}$, whether full time or part time. Within his

6 role, Simon often delivered coaching sessions himself across all age groups in the 7 academy.

8 Simon has accumulated twenty-five years of coaching experience in total. He 9 holds the UEFA Professional Licence (UEFA Pro), which is the highest coaching 10 qualification in Europe, and is only available to those working professionally at the 11 highest level. Simon also holds the Academy Managers Licence, for which the UEFA 12 Advanced Licence (UEFA A) is a prerequisite. Simon held the post of Head Coach for 13 a number of international youth teams, and was an Academy Manager at a club that 14 played in both the English Premiership and English Championship during his tenure.

The data collection process: diary-interview method

Data were collected from a work-based diary and four in-depth semi-structured interviews that amounted to around five and a half hours of conversation in total. During these meetings, Simon was encouraged to talk about his day-to-day work and reflect upon his experiences of managing the academy. During this process the diary captured Simon's everyday activities, where he engaged in a process of realityconstructing, meaning-making and identity work (Gibson, Mistry, Smith, Yoshida,

23 Abbott, \& Lindsay, 2013). The diary entries and interviews were flexible in nature, 24 allowing the freedom to explore issues that Simon felt were important. The solicited diary allows for the exploration of the intuitive internalized knowledge and logic which 
1 underpin the actions, interactions and meaning-making process in which Simon

2 engages every day (Gibson et al., 2013; Latham, 2003). Simon recorded specific

3 problems he encountered throughout his working day, along with the related emotions

4 and coping strategies utilised. The analysis of the diary revealed that Simon's role as

5 an English Premier League Academy Manager primarily involved coaching, managing

6 and dealing with a large number of unpredictable events, coping with ill-defined

7 problems as they arose and managing a large number of interrelated staff as he worked

8 towards the overall goals of 'the academy', and more widely 'the club'.

\section{Narrative data analysis}

11 The data were rigorously analysed using narrative thematic analysis in an 12 inductive (themes within the diary and interviews) and deductive (against sources of 13 managerial ambiguity and orchestration as an analytical frame) iterative process 14 (Smith, Bundon, \& Best, 2016). Narrative themes (patterns that run through a story) 15 were identified in Simon's data through a systematic coding process (Smith et al., 16 2016). During this process, analytic memos were developed to highlight tentative links between Simon's narrative and the analytical frame associated with orchestration, and they were used to help shape the questions and themes that were raised at the next interview as part of an iterative process (Smith \& Sparkes, 2002; Smith et al., 2016).

During this process, the second author acted as a 'critical friend', questioning

21 the initial interpretations of the data and suggesting alternative avenues to explore 22 (Smith \& Sparkes, 2002). In the following interviews Simon was asked to elaborate 23 upon previous discussions and invited to explore the relevant themes with the 24 interviewer, prompting a reflexive interviewing approach, specifically to explore the 25 how and why behind his coaching practices. In addition, because of his personal interest 
1 in and commitment to the research process, Simon agreed to read the interview

2 transcripts following each interview. He provided corrections and clarification of

3 intended meaning, and also commented upon the first author's interpretation of the data

4 through evolving drafts of the manuscript and the theoretical reading of Simon's

5 context. However, this was not considered to be a test of direct validation or truth

6 (Smith et al., 2014). Instead, it provided an opportunity for further reflexive

7 conversation between the participant and the first author (Smith et al., 2014). As

8 authors, we acknowledge that this is one potential reading of Simon's experiences as

9 an Academy Manager, and that the true complexity of his everyday experiences can

10 never be fully captured and represented in written text (Groom, Nelson, Potrac, \&

11 Smith, 2014; Sparkes, 1995). The representation offered here therefore cannot be 12 universal, definitive or final, and should instead be viewed as co-created in interactions 13 between the interviewer and interviewee, partially incomplete, contested, open to the 14 interpretation of others, storied and subjective (Smith et al., 2014; Sparkes, 1995).

\section{Judging interpretive case study research: towards naturalistic generalisation}

In following the ontological, epistemological and methodological logic of the interpretive case study, how then might we assess the quality, significance and contribution of the narratives presented? A number of criticisms and misunderstandings

20 of case study research continue to pervade the field. Flyvbjerg (2006) highlights that

21 within the social science literature, some authors have taken the position that you cannot

22 generalise from a single case, and therefore single case study research is viewed as less

23 valuable than hypothetico-deductive projects with a larger number of participants (i.e.

24 big- $N$ research seeking findings, which may be generalised to a larger population). 25 Flyvbjerg (2006, p. 220) has referred to the 'conventional wisdom of case-study 
1 research, which if not directly wrong, is so oversimplified as to be grossly misleading'.

2 Indeed, this suggests that 'general, theoretical (context-independent) knowledge is

3 more valuable than concrete, practical (context-dependent) knowledge' (Flyvbjerg,

4 2006, p. 221). However, in following the social science agenda (i.e. making the

5 invisible visible) within a complex, dynamic and socially negotiated field such as sports

6 coaching, there is no reason why this should be the case; indeed, on the contrary, it may

7 be argued that context-dependent knowledge might lead to more meaningful and

8 impactful work within the field.

9 Importantly, when judging the quality of contribution that such work may make

10 to the field, it is important that rich interpretive context-dependent work should be

11 judged within the paradigmatic (ontological, epistemological and methodological)

12 commitments of the research project. As opposed to the often perceived bias towards

13 verification, and lack of rigour of the interpretive case study has a number of

14 methodological strengths (Flyvbjerg, 2006). For example, unlike quantitatively based

15 large- $N$ research, case study research has the potential to 'close in on real life situations

16 and test views directly in relation to phenomena as they unfold in practice', which

17 forces researchers to revisit their initial understandings of the phenomena under

18 investigation (falsification) through engagement in the research process (Flyvbjerg,

19 2006, p. 235). Importantly, Ruddin (2006, p. 797) highlights that:

21 Case studies need not make any claim about the generalizability of their findings

22 but rather, what is crucial is the use others make of them - chiefly, that they

23 feed into the process of naturalistic generalization. (p. 804) 
Ruddin (2006) further highlights that within interpretive case studies, the test of naturalistic generalisation should be applied, in that "what is required of case study

3 research is not that they [the researchers] provide generalizations but rather, that they

4 illustrate the case they have studied properly, in a way that captures its unique features'

5 (p. 804). Therefore, when judging the quality of interpretive case study research and

6 the provision of contextually rich description, there is a 'realignment of the 7 responsibility to generalize away from the researcher towards the reader' (Ruddin, 8 2006, p. 804).

\section{Orchestration: An Analytical Frame}

Within the present study, the metaphor of orchestration was utilised as an analytical frame to highlight how through his interactions and negotiations with others,

13 Simon enacted his leadership and management roles in practice within the context of a

14 Premier League Academy. Wallace's (2003) concept of orchestration is grounded in

15 the recognition that 'it is probably beyond human capacity for any manager to achieve

16 fully predictable, directive control over the change process' (p. 9). Wallace (2004)

17 further identified that the metatask of orchestration (i.e. a task that deals with other 18 tasks and processes) entails instigating the plan, scope, strategy and financial 19 parameters of change, creating sustainable and favourable conditions for change to 20 happen, setting up management structures and delegating responsibility, monitoring the 21 process through regular feedback, and taking corrective action to keep the change 22 process on track.

Wallace $(2003,2004)$ developed the concept of orchestration through

24 examining the interactions that occur during complex organisational change in 25 educational settings. Orchestration is premised on the recognition of the early work of 
1 Hoyle (1986), that 'ambiguity is endemic to organisational life' and that 'people have

2 to work hard to make sense' of uncertainty (Wallace, 2003, p. 12). Wallace (2003)

3 identified three sources of ambiguity that constrain manageability during organisational

4 change: (1) individuals have variable but always limited control over other stakeholders

5 and their actions, (2) individuals have variable but limited awareness of what is

6 happening, and (3) contradictory values and beliefs are often held by individuals and

7 groups. Thus, the three generic sources of ambiguity act to limit manageability during

8 the process of complex organisational change, and they are often overlooked by

9 prescriptive rationalistic accounts of change management (Wallace, 2003).

10 Orchestration has been conceptualised as straddling both leadership and 11 management, as it is concerned with both 'making new things happen' and 'keeping 12 new things going' (Wallace, 2004, p. 62). Wallace (2004) defines orchestration as: system. (p. 58)

Wallace (2004) described the goal of orchestration as 'maintaining momentum and ensuring that the set course is followed' (p. 64). In this sense, orchestration reaches beyond the temporal dimensions of transformational leadership (i.e. the transformation of culture through charismatic, motivational leadership, stimulation and support)

23 towards long-haul leadership and management (Wallace, 2004). Indeed, whilst 24 orchestration is complementary to elements of transactional leadership (i.e. through 25 the promotion of the alignment of self-interest and reward), it reaches further towards 
1 bringing about complex change across multi-layered systems in the context of ongoing

2 work (Wallace, 2004). Wallace (2004) outlines three dimensions of orchestration

3 during complex change: (1) stages of the change process, (2) characteristics of

4 complexity, and (3) change management themes. The following section will briefly

5 outline each of the three dimensions.

\section{Stages of organisational change}

Wallace (2004) illustrated three stages in the change process. The first stage,

9 initiation, leads to the decision to instigate change within the organisation (Wallace, 10 2004). Following the decision to change, the second stage, implementation, is 11 concerned with the experiences of the orchestrator of change when putting the change 12 plans into action (Wallace, 2004). The final stage of the change process, 13 institutionalisation, refers to the way that the changes are seen as part of 'normal 14 practice' and no longer new (Wallace, 2004).

\section{Characteristics of complexity during the orchestration of organisational change}

Similar to the educational settings outlined by Wallace (2003, 2004), professional sports organisations are complex systems with a large number of individuals and groups of stakeholders (i.e. staff and players). Such groups are often organised into departments (technical staff, sports science and medical departments), and operate in an interrelated manner across system levels (i.e. age group related and employment status). Wallace (2004) identified five characteristics that typify the orchestration of organisational change.

Firstly, complex organisational change is large-scale (Wallace, 2004). For example, orchestrators of change must deal with a "multitude of stakeholders with a 
1 range of specialist knowledge and priorities' (Wallace, 2004, p. 68). Stakeholders often

2 hold 'partially incompatible beliefs and values', and have preferred 'alternative courses

3 of action' (Wallace, 2004, p. 68).

Secondly, that change is also likely to be componential, in that sequential and

5 overlapping components affect different stakeholders at different times (Wallace,

6 2004). Here, a 'multiplicity of differentiated but interrelated management tasks' need

7 to be addressed (Wallace, 2004, p. 69).

Thirdly, that complex change is systemic, where change within one part of the

9 system (e.g. departmental staffing resource change) influences other parts of the system

10 (e.g. change in cross-departmental support capabilities). Complex interactions occur

11 directly between individuals or through intermediaries and across system levels within 12 the organisation (Wallace, 2004). This, for example, includes face-to-face interactions 13 between stakeholders where unequal levels of social power between stakeholders and 14 system levels exist (Wallace, 2004).

Fourthly, complex change impacts stakeholders as differential impacting, with 'variable shifts in practice and learning required' (Wallace, 2004, p. 70). Over time, changes in practice will demonstrate 'variable congruence' to the desired change, and may be associated with stakeholders experiencing emotional responses to change 19 (Wallace, 2004, p. 70). Change will have 'variable' and at times 'reciprocal' effects 20 upon day-to-day activities within the organisation (Wallace, 2004, p. 70). Due to the 21 complexity of change, there will be "variable awareness of the totality of change beyond 22 those parts of immediate concern to particular individuals and groups' (Wallace, 2004, 23 p. 71).

Fifthly, that organisational change is contextually dependent, in that situational 25 factors and plans are influenced by other organisational and external concerns (Wallace, 
1 2004). For example, the financial resources that are available may result in

2 organisational change driving towards efficiency gains, large-scale investment in

3 capacity or downsizing the organisation (Wallace, 2004).

5 Change management and the orchestration of complex organisational change

6 There are three common themes associated with orchestration. The first is

7 flexible planning and coordination, covering management planning at different levels

8 of operation and over short-term, medium-term and long-term planning cycles

9 (Wallace, 2003, 2004). During this stage, the orchestrator (e.g. Academy Manager)

10 ensures that plans are monitored, coordinated and updated both informally and formally

11 in conjunction with the evolvement of the change process. The orchestrator may 12 delegate much of the planning detail to key staff with technical expertise (e.g. Heads of

13 Department - Head of Player Recruitment, Head of Medicine, Head of Sport Science,

14 Head of Performance Analysis, etc.).

15 The second theme developed by Wallace $(2003,2004)$ is culture building and

16 communication. This is achieved through the promotion of a culture of acceptance and

17 support, with consistent messages and feedback to assist coordination of change and

18 minimise resistance. During this phase, key staff are entrusted to apply the vision

19 outlined by the orchestrator to create the desired culture (Wallace, 2003). Often, senior

20 staff would articulate the vision to key stakeholders and emphasise the benefits of

21 change.

22 The third theme, differentiated support, encourages managers to accommodate

23 the differing needs of individual stakeholders (Wallace, 2003, 2004). The process of

24 identifying the individual needs is ongoing, with support ranging from forms of

25 education, training and continuing professional development to financial assistance. 
1 Here, the orchestrator of change would seek to ensure that the staff have the necessary

2 skills and support to enact the required changes.

3

\section{Results and Discussion}

Through the interviews with Simon and subsequent analysis of the setting, characters and plot in Simon's narrative, we came to understand that the central feature of the story that Simon shared with us was that his primary role was to create organisational change within the academy. As such, the following section follows five interrelated themes: (1) a vision for organisational change, (2) problems implementing organisational change, (3) managing staff ambiguity, (4) strategies for organisational change, and (5) supporting the change process.

\section{A vision for organisational change}

A key feature of Simon's narrative was that he was appointed to facilitate organisational change. Simon explained that when initiating change within a football academy 'you have to be pretty clear in terms of your vision and how you are going to play and what your style is going to be'. Simon described his initial remit in the following way:

I was appointed because of what the club [directors] felt was needed. I presented my ideas, and they [club directors] let me know their perception of the academy. I told them my perception, and I was then given carte blanche to implement that. They entrusted me. I had a brief, but I also briefed them about what I stood for and believed in. 
Following Simon's appointment to the club, and with the backing and support

2 of the board of directors, he outlined his future vision for the club's academy. In his

3 words:

4 17

I had a vision of how we were going to play, and when I came in, I looked at the coaches here who were able to grasp that. Some could, some disagreed, some had to move on. So, if you were the Under 12 coach and you got upset, then so be it, you would go. It's not your team, it's an Athletic FC [pseudonym] team and I'm going to insist on the senior and best coaches working with them and you would join in and learn. And then you're constantly looking to bring people in who empathise with what you're doing and can see the same pictures that you're seeing along the way. And what you're trying to do with the coaches is educate them.

In further describing the thoughts and emotions that he experienced during this period Simon highlighted that:

The club decided something wasn't right and it needed changing, but it was difficult because I was on my own. Every time you're having meetings where 0 people have to move on, or you're making harsh decisions on people's lives, it's 1 very uncomfortable to sleep the night before. Without being a cynical git, I think that is going to be the same in any business that looks to try and change things. 
Similarly, during the change process Simon described his understanding of the context of elite football and the impact of the change on some of the staff within the academy:

4 They've been people who have been put out of a job; I think it's only human nature that that's going to happen. I think that's natural in highly competitive organisations like in football and academies where everyone's looking for people to fail.

Simon's meeting with the club directors to discuss the need for change within the academy is the first stage in the change process, initiation, deciding to "proceed with change' (Wallace, 2004, p. 72). Here, Simon highlighted his perceptions of the academy as an outsider and the need for a change of philosophy in the academy. Simon explained that 'in the old way they were getting success, but in my view it was shortterm success. They were not evolving to Champions League [elite European club competition] players.'

Following the decision to initiate change, Wallace (2004) describes implementation as the experience of 'attempting to put change into practice' (p. 72). The strategies employed by Simon when implementing his philosophy are in line with the first theme of orchestration, described by Wallace (2003, p. 25) as 'flexible planning and coordination', particularly when looking at the staff coaching the different age groups who 'were able to grasp' the philosophy being imposed. Such preparation by managers needs to ensure 'short-term flexibility through incremental planning and coherence through longer planning cycles' (Wallace, 2003, p. 25). Furthermore, Simon described the 'highly competitive' nature of football, where 'everyone's looking for 
1 people to fail'. This illustrates a key characteristic of the complexity within which

2 Simon operates. That is, elite youth football can be viewed as a highly context-

3 dependent environment (Wallace, 2003, 2004), with its own deeply embedded socio-

4 cultural practices and norms (Cushion \& Jones, 2006, 2012; Groom et al., 2012).

5

6 Problems implementing organisational change

Simon shared with us that the process of implementing organisational change

8 was far from straightforward and unproblematic. He provided the following example

9 of an interaction with a first team coach at Athletic FC, illustrating the difficulty of

10 trying to make long-term changes for the benefit of the club and how they can be viewed

11 negatively from a short-term perspective:

He said "if you were First Team Manager and lost 7-2 you would have been

When prompted on his feelings during these initial periods of change he explained: sacked", but so long as you're strong in your vision and defend your beliefs, especially if they're different to other people, you deal with it.

I think it's only human nature that it is going to happen. It can be pretty claustrophobic around the club where maybe you've had a bad week or a couple of knockbacks. Because you're stretching kids fully to the limit, you're going to have short-term failures, and you need these failures to have success. 
Simon encountered a number of problems along the way that required him to year. 11 列

Originally, I did it [implemented change] through presentations, giving them handouts and saying, 'this is what I want you to do'. But I realised I can't sit you down and say, 'here's a session, go and do that'. You've got to be able to see it, feel it, and know where you're going. For example, we've just brought in Geoff. He's played the game, played under Mike [pseudonym]. He speaks the language; when you speak to him he understands it. When you interact with him he'll say something and you'll know he's on the right lines.

When implementing organisational change, Simon had to deal with resistance to change from elements of both coaching staff and players. The first example highlights a situation where the players reported that coaches had 'reverted back to type' during training sessions, when Simon was not present to observe and monitor practice in person:

The players told me what happened. I think they believed in what we were doing more than some of the coaches did. So I addressed the coaches directly, and if you got the sense that this is something that they didn't believe in then we would have to agree to disagree and they moved on because they were not able to ply their trade how we wanted it. And that happened over a period of months and a 24 
In another incident following his attempts to change the culture of the academy,

2 Simon described a situation where he had received resistance from the players:

3

Quite a few of the players [U18s] were very, very disrespectful towards our sport scientists. We do these osmos [physiological test of urine to measure hydration and readiness to train] and they started putting water in them. We played a game yesterday and they were dehydrated so, as staff, we decided that on this Monday we would remove the 'gold standard' support that they get and gave it to the U16s. So the U18s trained at a different place, we deliberately did the most linear, boring session that could ever be invented with one flat ball, and they had no debrief afterwards. If they weren't prepared to accept all these marginal gains, then fine. It was thought out to try and get them to understand what they've actually got and not to take things for granted. It's rewriting a computer; it couldn't be done in a year, and it couldn't be done in three years.

Simon provided a number of additional examples of the cultural discourse of professional football and the context within which he attempted to orchestrate change. In particular, the explicit discourse of results related to 'sacking' due to perceived 'short-term failures' was normalised and permeated through the club (Cushion \& Jones, 2006, 2012). Simon realised that the communication of his vison for the club through traditional means (i.e. staff presentations) was ineffective in creating change. Wallace (2003) highlights that 'since managers depend on stakeholders to accept and implement change, they must forge a culture of acceptance for their vision' (p. 25). Simon described this process as 'rewriting a computer.' In trying to build the desired culture and communicate the new vision to the staff, Simon realised that the staff had to 'see 
1 it, feel it, and know where you're going'. In attempting to communicate this cultural change, Simon employed key members of staff who could 'speak the language'. However, during the process of implementing organisational change Simon

4 encountered resistance from both the staff and some of the senior youth players

5 'reverting back to type'. During the process of organisational change, contradictory

6 beliefs and values may become evident and lead to situations of conflict between 7 individuals and groups (Wallace, 2003). Whilst communicating consistent messages

8 and gathering feedback can be used to pre-empt resistance (Wallace, 2004), using 9 sanctions such as removing the 'gold standard' sport science support from the U18 team 10 highlights the quest for stakeholder [player] buy-in to the new values and beliefs of day11 to-day working practices (Santos et al., 2013). Wallace (2003) states that the task of managing such resistance relies on methods of surveillance and sanctions. This was

13 evident in Simon's story, in situations where the coaches were not meeting his standards 14 or they were practising in a way that was opposed to the new vision. During such periods 15 of resistance and the exercise of individual human agency by the coaches, Simon used 16 his own agency (i.e. his capacity to act independently) in an attempt through persuasion 17 to channel others' agency towards his vision of change, whilst monitoring and providing 18 corrective action delimiting the agency of those who did not embrace change (Wallace, 19 2004). When discussing his interactions with those who were unable to or refused to 20 adapt to the change process, Simon explained that 'we would have to agree to disagree 21 and they moved on'. Therefore, the structural limits (i.e. what is do-able/un-do-able and 22 what is thinkable/unthinkable) that Simon imposed on the change process were enacted 23 to constrain the individual agency of those who resisted (Wallace, 2004). Within a 24 sporting setting, as Jones and Wallace (2005, p. 24) further explain, 'orchestration 25 involves monitoring others' practice relating to the change, channelling their agency in 
1 the desired direction through encouragement and incentives, and attempting to delimit

2 their agency through the threat of corrective action where their practice is deemed

3 unacceptable.'

\section{Managing staff ambiguity during change}

In elaborating upon his experiences during the initial period of change, it became evident that Simon had to manage ambiguity within the academy. Simon explained that:

9
There are certain things that are happening or evolving and I don't know what the answer is going to be. I have one member of staff that is always worrying, you know, 'what is going to happen here?' I don't know, I haven't got a crystal ball. You have to go with it, ride the punches, and suss it out as it evolves. Some staff find it hard to grasp that I don't know what the answers are. We have this new Chief Executive coming in, what does he want? What does he want to do with this? I don't know. If he wants ' $x$ ', this is how we will deal with it, if he wants ' $y$ ', this is how we will deal with it, if he wants ' $z$ ', well, then we will think about it. The book doesn't exist that says 'here's the problem, this is the answer'; you have to think on your feet, and I'm sure that's the same whether you're manager of Tesco or a Premier League Academy Manager. necessary:

In attempting to manage ambiguity and prevent uncertainly and anxiety within the academy staff, Simon highlighted that he would only pass on information when 
Let's say there is a concern going on, I would rather take the worry myself. I wouldn't say 'I've heard $\mathrm{x}, \mathrm{y}$ and $\mathrm{z}$ about finances here". I would let people know on a need-to-know basis that there might be future changes I am thinking about, but I wouldn't just throw them in and worry people because I don't know the answer. (1)

Simon explained that organisational ambiguity was endemic in his role, and that he often did not know 'what is going to happen' because he did not have a 'crystal ball'. Ambiguity within organisational life can stem from 'opaqueness connected with internal decision-making' (Wallace, 2003, p. 12). Prevalent sources of ambiguity for Simon were his limited control and limited awareness of other stakeholders' actions (e.g. the Chief Executive). Wallace (2003) identifies limited control as the principal source of ambiguity that constrains manageability. In particular, Simon highlighted that 'The book doesn't exist that says "here's the problem, this is the answer".' Wallace (2003) further explained that individuals always have 'equally variable but always limited awareness of what is happening' during the change process (p. 12). Wallace (2003) highlights that organisational change increases ambiguity as it 'challenges habitual practices and beliefs of the people around the organisation' and is associated with new experiences and new learning. Similarly, Potrac and Jones (2009 p. 570) highlighted how the 'introduction of changes in working practices can produce dissonance within an organisation', and therefore it should be managed carefully with thought given to the consequences of change for those affected. Such change increases the potential for unintended consequences among stakeholders within the organisation, as change is exercised in a componential and systemic manner across different system levels and in a sequential and overlapping manner, affecting different individuals at 
1 different times, thus increasing unpredictability (Wallace, 2003, 2004).

Strategies for organisational change

One of the strategies that Simon employed to initiate organisation change was to use senior coaches in strategic ways to work with and communicate his messages to

6 stakeholders throughout the academy. Here, he explained that:

7
I nicked one of Mike's [pseudonym, managed over 1,500 professional football games] ideas really. I delegated one of my best coaches and removed all of his administration off him and insisted that he worked with all the players and coaches. Therefore, the people who were driving it forward knew what it looked like in the end and believed passionately in it. We all helped each other when it wasn't working and I think the stubbornness, it sort of permeates down to the lower teams. It didn't give any opportunities for part-time people who didn't necessarily believe in it, it didn't give them time to say, 'Ah we're not doing that, we're doing it our way', because there were always full-time members of staff down there, so that we'd never let that happen. So the message was permeating down on a daily basis. There was always someone there who strongly believed in what we were doing, drilling it down, not really letting any cancers develop in what we were trying to do.

In an attempt to implement a new 'style', Simon developed language and terminology using current, well-known professional football players' names and their best attributes and related them to movements, tactics and techniques specific to his academy: 
Often we will refer to a “[Fernando] Torres" run, or a "[Cristiano] Ronaldo" or "[Zinedine] Zidane" who can dominate with his back to goal, or your "[Marc] staff' by providing a clear plan and vision for the future. The strategies employed by Simon in scrutinising the coaches throughout the academy are consistent with previous work on the activities of an orchestrator. Wallace (2004) highlights that senior staff can be utilised to create favourable conditions for change through setting up management structures and delegating responsibility, monitoring progress and taking 'corrective or adaptive action to keep the change process on track' (p. 66). Here, Simon entrusted key staff to apply his vision to create the desired culture where stakeholders 'believed passionately in it' (Wallace, 2003). During this process senior staff would often

21 articulate the vision to key stakeholders and emphasise the benefits of change. In

22 Simon's words, they 'strongly believed in what we were doing, drilling it down, not

23 really letting any cancers develop in what we were trying to do' in an attempt to 'pre-

24 empt any resistance' (Wallace, 2003, p. 25). Here, the large-scale characteristics of 25 complexity of change across multiple stakeholders, with 'partially compatible beliefs 
1 and values' and with 'preferred courses of action', are evident in Simon's narrative

2 (Wallace, 2004, p. 68). In particular, Simon was aware of potential gaps in practices

3 appearing between full-time and part-time staff.

Simon developed a 'language' within the club for culture building and

5 communication, the second theme of orchestration, that senior coaches understood and

6 would use to increase the players' understanding of what was required. Simon achieved

7 this by using well-known professional football players as role models to discuss role

8 related performance (Wallace, 2003).

\section{Supporting the change process}

Simon quickly identified the importance of supporting his staff to 'be the best that they could be' with 'a thirst for knowledge and personal and professional development'. Indeed, Simon actively encouraged his staff to attend as many

14 educational and training courses as possible and to take opportunities to learn from other elite sports environments:

If we are to say to the kids that they have to have the desire to improve, then that has to be a significant trait of the staff as well. I want them to always be looking for something better, telling me, 'I want to go on this course', 'I want to go on that course', or 'I want to go up to United for a week to learn and look at what they do'. Then my job is to try to facilitate that.

Simon offered an insight into the importance of providing different aspects of 24 support to both coaches and players in managing the change process and implementing his philosophy. Initially, Simon identified the need to educate the coaches working 
1 within the academy and revolutionise their way of thinking. To effect such change,

2 Simon allocated his top coach to work constantly with the other coaches of different

3 age groups, providing a level of support, as previously discussed: 'His job was to work

4 evenings and weekends, so he would educate and mentor coaches on a daily basis and

5 lead by example.' Subsequently, the coaches would then observe best practice and 'join

6 in, and learn'. Simon provided a deeper insight into the support afforded to coaches to

7 ensure that they were equipped to deliver a change in practice and philosophy:

8

9 Originally, when I came in all the preparation had been done before. I came in and let people know it was going to be done this way and why. What we were trying to do was produce players capable of playing in the Champions League. So we had to equip our boys with the skills to be able to compete with players from all around the world, so we had to be different from other clubs. So they were told why we were going to do it and what they were going to do. Then we would support them by doing in-service training. That had a minimal effect in terms of power points, it might have a 24 -hour effect... but... what we then did was change one member of staff who believed in what we were doing and fully absorbed what we were doing and then he became in charge of all player development. His job was to work with the players on a daily basis and work across all age groups. So rather than telling, they were being shown what to do. Then I would work up there myself on a regular basis and take a team on a Sunday.

Simon elaborated further to outline the methods and strategies he employed to ensure that coaches were practising in a manner that would enhance the development 
1 of players in line with the philosophy of the academy. Simon stated that:

2

If there is a senior, more experienced coach about then they will take the session. You cannot be offended by that; it's not trying to undermine what you are doing or undervalue what you are doing. What's really important is that the senior coaches know all of the players. You can't be offended because the senior coach is probably a more experienced coach. It also gives the other coaches the chance to get ideas down and learn off the senior coaches. And I think that the others are accepting that now. I'll try and engender that atmosphere where they will stay behind and maybe watch the youth team train, or maybe if they finish at seven they will stay until eight and watch Michael or me or Jack work. I was in there the other night until 9 o'clock because two coaches wanted to know more about the session and had loads of questions. It was a genuine interest in them wanting to get better. There are one or two that see it as a threat and we have to work on them and say, 'hang on, why are you working with twenty kids and your assistant is putting cones out? Put your cones down and you can both work with 10.'

Simon discussed that one of his roles in supporting organisational change was to ensure that the staff had access to and support for their own development. Wallace suggests that during change, differentiated support, the third theme of orchestration, is required to accommodate the differing needs of staff (Wallace, 2003, 2004). For example, the process of identifying ongoing individual needs and support ranges from education, training and continuing professional development to financial assistance. Through a process of 'in-service training' and delegation of responsibilities to key members of staff (i.e. 'all player development'), Simon was able to 'show' rather than 
1 'tell' staff how he wanted them to fulfil their roles by being able to 'work up there

2 myself on a regular basis'. Here, Simon's role is to ensure that the staff have the

3 necessary skills and support to enact the required changes. This is because complex

4 organisational change is differentially impacting, with 'variable shifts in practice and

5 learning required' (Wallace, 2004, p. 70).

6 Due to the complexity of the change process, Simon utilised senior coaches to 7 support the development of other coaches within the academy. Simon highlighted that

8 whilst he wanted the senior coaches to get to know the players, he also wanted the less

9 experienced coaches to have the opportunity to learn from senior mentors. Through this 10 process, change had a 'variable' and at times 'reciprocal' effect upon day-to-day 11 activities within the organisation (Wallace, 2004, p. 70). In addition, over time, changes 12 in practice demonstrated 'variable congruence' to the desired change, and may be 13 associated with stakeholders experiencing emotional responses to change (Wallace, 14 2004, p. 70). Simon highlighted that insisting senior coaches were able to lead sessions 15 initially led to some resistance but that he attempted to create and 'promote a culture of 16 acceptance and support' where this became the accepted norm and culture in the club 17 (Wallace, 2004, p. 67). This relates to Wallace's (2004) final stage of the change, 18 institutionalisation, where the perception is of 'normal practice and so no longer 19 anything new' (p. 72). Simon's work with the coaches to embrace the culture change 20 'as the norm', for example through his interactions with coaches staying behind to 21 discuss the session, reflects the atmosphere that he had wanted to create to ensure the 22 longevity of change.

\section{Conclusion}


1 League Academy Manager through the process of organisational change. Through

2 Simon's narrative we identified the utility of the three stages of change identified by

3 Wallace (2004) to understand Simon's actions, and the motives behind the strategies

4 that were implement to affect organisational change. Specifically, the initiation stage

5 highlighted a need for change within the academy and the playing philosophy attached

6 to the academy. The implementation stage portrayed Simon's strategies to manage a

7 change in philosophy and the day-to-day working practices of the academy to ensure

8 that the new philosophy was being enforced. Finally, within the institutionalisation

9 stage the changes implemented by Simon became the norm within the academy and the 10 working practices of the coaches.

11 Additionally, the notion of orchestration (Wallace, 2003) as an analytical frame 12 allowed for a deeper comprehension of Simon's experiences of managing complexity 13 and organisational change. The first theme highlighted was the need for flexible 14 planning and coordination (Wallace, 2003). In this instance, Simon discussed the 15 importance of understanding the philosophy of the academy and identifying the coaches 16 who were on board with that philosophy and those who were not. The second theme 17 was the process of culture building and communicating this culture effectively to staff 18 and coaches (Wallace, 2003). Here, Simon indicated that he had to be acutely aware of 19 the increase in specialist staff working in a Premier League Academy and how this 20 increase could prompt factions and disagreements to emerge and act as negative forces 21 against the process of change. Finally, the importance of providing differentiated 22 support to accommodate the needs of individuals during this change process became 23 apparent (Wallace, 2003).

24 Whilst we recognise the limitations of a single-participant case study design, 25 we believe that the rigorous data collection and the analysis presented here offer new 
1 insights into coaching practice. Specifically, we believe that this paper further builds

2 empirical support for the notion that the everyday realities that sports coaches face

3 primarily involve managing ambiguous complex social situations (Santos et al., 2013).

4 To date such an appreciation is not reflected in the way that we educate sports coaches

5 to understand and prepare for the realities of practice (Jones, Morgan, \& Harris, 2012;

6 Chesterfield, Potrac, \& Jones, 2010).

From an analytical perspective, we also believe that orchestration offers a

8 flexible way to look at complexity, one that does not require events to be de-humanised

9 and rationalistically reduced and simplified, and devoid of the essential 'messy' nature

10 of human endeavour. Wallace (2003) identified the benefits of undertaking projects

11 immersed in knowledge-for-understanding and instrumentalism in 'seeking more 12 realistic ways of supporting practitioners with managing complex educational change 13 inside the limits of human agency' (p. 28). As opposed to concentrating on notions, 14 suggesting agency to be potentially unlimited, Wallace (2003) contended that 15 knowledge-for-action is best served by developing coping strategies underpinned by 16 concepts such as orchestration. The development of effective coping emphasises the 17 improvement in managing ambiguity and accepting that a certain level of ambiguity is 18 inherent within managerial life (e.g. Wallace, 2003; Jones \& Wallace, 2005; Bowes \& 19 Jones, 2006).

\section{Suggestions for future work: methodological and practical considerations}

From a methodological perspective, nested case studies could be used (i.e.

23 examining the effects upon interrelated key individuals) to further explore the impact

24 of reciprocal, sequential and overlapping change, both within and between system 25 levels. Thomas (2011, p. 517) suggests that 'a nested case study is distinct from a 
1 straightforwardly multiple study in that it gains its integrity, its wholeness from the

2 wider case', allowing for an interpretation of the intertwined relationships within each

3 case. This methodological approach would allow for a comparative relational

4 understanding of the interactions from the perspectives of multiple interrelated actors

5 within the coaching environment to further a poly-vocal account of organisational life

6 in coaching contexts (Potrac, Nelson, \& O'Gorman, 2015).

From a practical perspective, one way that the findings of this study might be

8 used in future coach education programmes is through novel student-focused

9 pedagogical practices. For example, within the sports coaching literature, problem-

10 based learning (PBL) (Jones and Turner, 2006) and ethno-drama (ED) (Morgan, Jones,

11 Gilbourne and Llewellyn, 2013) have been utilised to create contextual, complex, 12 dynamic and realistic learning situations supported by subtle tutor interaction and 13 questioning. Whilst PBL focuses upon realistic, problematic scenarios and subtle tutor 14 questioning (Jones and Turner, 2006), ED centres on problems presented through 15 dramatic performance in order to provide solutions to those problems, which often 16 escalate in complexity as more information becomes available through performances 17 (Morgan et al., 2013). When applying the findings of this study to such approaches, 18 coach educators might wish to consider building realistic scenarios that place the 19 coaches/managers in situations that they might encounter within a football 20 environment. For example, a scenario based upon implementing organisational change 21 within 'the club' might be used to generate dialogue between student-managers to 22 facilitate learning. Here, students might be provided with contextual information about 23 the club, with the specific goals and performance targets set by the board (e.g. specific 24 targets regarding the number of players progressing into the first team, youth and senior 25 international player production, sales and financial viability of the club's transfer 
1 market activities, etc.) and the wider organisational concerns (e.g. the development of

2 the club's strategy, philosophy and identity). Students could be encouraged to discuss

3 how they would assess, plan, implement and support change within the club. Through

4 subtle tutor questioning and realistic problematic disruptions (e.g. unplanned meetings

5 and presentations to senior executives at boardroom level), students would be

6 encouraged to give thought to and develop the knowledge and skills required to operate

7 in senior coaching management roles during the change process.

8 Alternatively, utilising an ED approach, students might watch a dramatic

9 performance of a dialogue between a Chief Executive and an Academy Manager in a

10 job interview or view the first team manager undertaking a media interview to explain

11 why they have so few academy players playing for the team, and then consider how the 12 new Academy Manager (the student) has been tasked by the club to address this issue 13 and the expectations. Students might then have to prepare to give a media conference 14 themselves, to share their ideas around implementing change within the club.

\section{Acknowledgements}

17 We would like to thank two anonymous reviewers, and Dr Lee Nelson and Professor 18 Paul Potrac, for their valuable feedback and suggestions on earlier drafts of this paper. 19 Any errors that remain are our own.

\section{References}

Bowes, I., \& Jones, R.L. (2006). Working at the edge of chaos: understanding coaching as a complex, interpersonal system. The Sport Psychologist, 20, 235245.

25 Chesterfield, G., Potrac, P., \& Jones, R.L. (2010). 'Studentship' and 'impression 
management' in an advanced soccer coach education award. Sport, Education \& Society, 15, 299-314.

3 Cruickshank, A., \& Collins, D. (2012a). Change management: The case of elite sports performance. Journal of Change Management, 12(2), 209-229.

5 Cruickshank, A., \& Collins, D. (2012b). Culture change in elite sport performance teams: Examining and advancing effectiveness in the new era. Journal of Applied Sport Psychology, 24, 338-355.

Cruickshank, A., \& Collins, D. (2012c). 'Multi-directional management': Exploring the challenges of performance in the World Class Programme environment. Reflective Practice, 13(3), 455-469.

Cruickshank, A., Collins, D., \& Minten, S. (2014). Driving and sustaining cultural change in Olympic sport performance teams: A first exploration and grounded theory. Journal of Sport and Exercise Psychology, 36(1), 107-120.

Cushion, C., \& Jones, R.L. (2006). Power, discourse and symbolic violence in professional youth soccer: the case of Albion F.C. Sociology of Sport Journal, $23,142-161$.

Cushion, C., \& Jones, R.L. (2012). A Bourdieusian analysis of cultural reproduction: socialisation and the 'hidden curriculum' in professional football. Sport, Education \& Society, 19(3), 276-298.

Flyvbjerg, B. (2006). Five misunderstandings about case-study research. Qualitative Inquiry, 12(2), 219-245.

Gibson, B.E., Mistry, B., Smith, B., Yoshida, K., Abbott, D., \& Lindsay, S. (2013). The integrated use of audio diaries, photography, and interviews in research with disabled young men. International Journal of Qualitative Methods, 12, $382-402$. 
1 Groom, R., Cushion, C., \& Nelson, L. (2012). Analysing coach-athlete 'talk in interaction' within the delivery of video-based performance feedback in elite youth soccer. Qualitative Research in Sport, Exercise \& Health, 4(3), 439458.

Groom, R., Nelson, L., Potrac. P., \& Smith, B. (2014). Writing and representing research. In L. Nelson, R. Groom, \& P. Potrac (Eds.), Research methods in sport coaching (pp. 86-97). London: Routledge.

Hoyle, E. (1986). The politics of school management. London: Hodder \& Stoughton.

Jones, R.L., Bailey, J., \& Thompson, I. (2013). Ambiguity, noticing, and orchestration: Further thoughts on managing the complex coaching context. In P. Potrac, W. Gilbert, \& J. Denison (Eds.), The Routledge handbook of sports coaching (pp. 271-283). London: Routledge.

Jones, R.L., \& Corsby, C. (2015). A case for coach Garfinkel: decision making and what we already know. Quest, 67(4), 439-449.

Jones, R.L., Morgan, K., \& Harris, K. (2012). Developing coaching pedagogy: seeking a better integration of theory and practice. Sport Education \& Society, 17(3), 313-329.

Jones, R.L., \& Turner, P. (2006). Teaching coaches to coach holistically: can Problem-Based Learning (PBL) help? Physical Education and Sport Pedagogy, 11(2), 181-202.

Jones, R.L., \& Wallace, M. (2005). Another bad day at the training ground: coping with ambiguity in the coaching context. Sport, Education \& Society, 10, 119134. 
1 Latham, A. (2003). Research, performance, and doing human geography: Some reflections on the diary-photography, diary-interview method. Environment and Planning A, 25(11), 1993-2017.

Morgan, K., Jones, R.L., Gilbourne, D., \& Llewellyn, D. (2013). Changing the face of coach education: using ethno-drama to depict lived realities. Physical Education and Sport Pedagogy, 18(5), 520-533.

Nelson, L., Potrac, P., Gilbourne, D., Allanson, A., Gale, L., \& Marshall, P. (2013). Thinking, feeling, acting: the case of a semi-professional soccer coach. Sociology of Sport Journal, 30, 467-486.

Potrac, P., \& Jones, R.L. (2009). Micropolitical workings in semi-professional football. Sociology of Sport Journal, 26, 557-577.

Potrac, P., Jones, R., Gilbourne, D., \& Nelson, L, (2013). Handshakes, BBQs, and bullets: A tale of self-interest and regret in football coaching. Sports Coaching Review, 1, 79-92.

Potrac, P., Nelson, L.N., \& O'Gorman, J. (2015). Exploring the everyday realities of grassroots football coaching: towards a relational perspective. Soccer and Society. DOI: 10.1080/14660970.2015.1100900

Relvas, H., Littlewood, M., Nesti, M., Gilbourne, D., \& Richardson, D. (2010). Organizational structures and working practices in elite European professional football clubs: Understanding the relationship between youth and professional domains. European Sport Management Quarterly, 10(2), 165-187.

Ruddin, L.P. (2006). You can generalize stupid! Social scientists, Bent Flyvbjerg, and case study methodology. Qualitative Inquiry, 12(4), 797-812. 
1 Santos, S., Jones, R.L., \& Mesquita, I. (2013). So coaches orchestrate? The working practices of elite Portuguese coaches. Research Quarterly for Exercise \& Sport, 84, 263-272.

Smith, B. (2013). Sporting spinal cord injuries, social relations, and rehabilitation narratives: an ethnographic creative non-fiction of becoming disabled through sport. Sociology of Sport Journal, 30, 132-152.

Smith, B., Bundon, A., \& Best, M. (2016). Disability and activist identity: a qualitative study of narratives of activism among elite athletes with impairment. Psychology of Sport and Exercise, 26, 139-148.

Smith, B., \& Sparkes, A.C. (2002). Sport, spinal cord injury, embodied masculinities, and the dilemmas of narrative identity. Men and Masculinities, 4, 258-285.

Smith, B., \& Sparkes, A.C. (2009). Narrative analysis and sport and exercise psychology: understanding lives in diverse ways. Psychology of Sport and Exercise, 10, 279-288

Smith, B., \& Sparkes, A.C. (2014). Qualitative research methods in sport, exercise and health: from process to product. London: Routledge.

Smith, B., Sparkes, A.C., \& Caddick, N. (2014). Judging qualitative research. In L. Nelson., R. Groom., \& P. Potrac (Eds.), Research methods in sport coaching (pp. 192-201). London: Routledge.

Spall, S. (1998). Peer debriefing in qualitative research: Emerging operational models. Qualitative Inquiry, 4(2), 280-292.

Sparkes, A.C. (1995). Writing people: reflections on the dual crises of representation and legitimation in qualitative inquiry. Quest, 47, 158-195.

Thacher, D. (2006). The normative case study. American Journal of Sociology, $111(6), 1631-76$. 
1 Thomas, G. (2011). A typology for the case study in social science following a review of definition, discourse and structure. Qualitative Enquiry, 17(6), 511-521.

3 Thompson, A., Potrac, P., \& Jones, R.L. (2015). 'I found out the hard way':

4 5 micropolitical workings in professional football. Sport, Education \& Society, 20, $1-19$.

6 Wallace, M. (2003). Managing the unmanageable?: coping with complex educational 7 change. Education Management Administration Leadership, 31, 9-29.

8 Wallace, M. (2004). Orchestrating complex educational change: local reorganisation 9 of schools in England. Journal of Educational Change, 5, 57-78. 